\title{
Metrics for Public Health Perspective Surveillance of Bacterial Antibiotic Resistance in Low- and Middle-Income Countries
}

Olga Tosas Auguet $^{1 *} ¥$, Rene Niehus ${ }^{2} ¥$, Hyun Soon Gweon ${ }^{3,4}$, James A. Berkley ${ }^{1,5,6}$, Joseph Waichungo ${ }^{5}$, Tsi Njim ${ }^{1}$, Jonathan D. Edgeworth ${ }^{7}$, Rahul Batra ${ }^{7}$, Kevin Chau ${ }^{8}$, Jeremy Swann ${ }^{8}$, Sarah A. Walker ${ }^{8,9}$, Tim E. A. Peto ${ }^{8,9}$, Derrick W. Crook ${ }^{8,9}$, Sarah Lamble $^{10}$, Paul Turner ${ }^{1,11}$, Ben S. Cooper $^{1,12}$, Nicole Stoesser ${ }^{8,9}$

${ }^{1}$ Centre for Tropical Medicine and Global Health, University of Oxford, Oxford, UK

${ }^{2}$ Harvard T.H. Chan School of Public Health, Harvard University, Boston, USA

${ }^{3}$ School of Biological Sciences, University of Reading, Reading, UK

${ }^{4}$ Centre for Ecology \& Hydrology, Wallingford, UK

${ }^{5}$ KEMRI/Wellcome Trust Research Programme, Kilifi, Kenya

${ }^{6}$ The Childhood Acute Illness and Nutrition (CHAIN) Network, Nairobi, Kenya

${ }^{7}$ Centre for Clinical Infection and Diagnostics Research (CIDR), Department of Infectious Diseases, King's College London, London, UK

${ }^{8}$ Nuffield Department of Medicine, University of Oxford, Oxford, UK

${ }^{9}$ NIHR Health Protection Research Unit in Healthcare-associated Infections and Antimicrobial Resistance, UK

${ }^{10}$ Wellcome Trust Centre for Human Genetics, University of Oxford, Oxford, UK

${ }^{11}$ Cambodia-Oxford Medical Research Unit, Microbiology Department, Angkor Hospital for Children, Siem Reap, Cambodia

${ }^{12}$ Mahidol-Oxford Tropical Medicine Research Unit, Faculty of Tropical Medicine, Mahidol University, Bangkok, Thailand

* Corresponding author

${ }^{¥}$ Joint first authors

\section{Abstract}

Antimicrobial resistance (AMR) is a global health threat, especially in low-/middle-income countries (LMICs), where there is limited surveillance to inform empiric antibiotic treatment guidelines. Enterobacterales are amongst the most important causes of drug-resistant bacterial infections. We developed a novel AMR surveillance approach for Enterobacterales by profiling pooled human faecal metagenomes from three sites ( $\mathrm{n}=563$ individuals; Cambodia, Kenya, UK) to derive a taxonomyadjusted AMR metric ("resistance potential") which could be used to predict the aggregate percentage of resistant invasive Enterobacterales infections within each setting. Samples were sequenced (Illumina); taxonomic and resistance gene profiling was performed using ResPipe. Data on organisms causing bacteraemia and meningitis and antibiotic susceptibility test results from 2010-2017 were collated for each site. Bayesian generalised linear models with a binomial likelihood were fitted to determine the capacity of the resistance potential to predict AMR in Enterobacterales infections in 
each setting. The most informative model accurately predicted the numbers of resistant infections in the target populations for 14/14 of antibiotics in the UK, 12/12 in Kenya, and 9/12 in Cambodia. Intermittent metagenomics of pooled human samples could represent a powerful pragmatic and economical approach for determining and monitoring AMR in clinical infections, especially in resource-limited settings.

\section{Introduction}

Antimicrobial resistance (AMR) is a global health emergency ${ }^{1}$, and imposes a particularly large socioeconomic burden in resource-limited settings, where bacterial infections and several other drivers of AMR commonly co-occur and effective antibiotics may be unavailable or unaffordable ${ }^{2}$. A key pillar in AMR mitigation is the development of effective and standardised AMR surveillance, to monitor trends, inform empiric treatment guidelines, identify emerging AMR threats, and monitor the impact of interventions. There has been significant investment in surveillance capacity, such as by the UK's Fleming Fund, and an attempt to promote standardised collection, analysis and sharing of global AMR data with an emphasis on capturing clinical and microbiological information, encapsulated in the WHO Global Antimicrobial Resistance Surveillance System (GLASS) ${ }^{3}$. However, limitations in implementing GLASS include the time taken to develop robust infrastructural capacity to support data collection in regions where AMR is most relevant or prevalent, and the difficulty in obtaining systematic datasets even from enrolled countries with adequate infrastructure, especially outside tertiary or University centres. Surveillance strategies which could bridge or complement the implementation of approaches such as GLASS would be helpful.

Colonisation with specific species and/or drug-resistant organisms, such as nasal colonisation with Staphylococcus aureus ${ }^{4}$, or rectal colonisation with carbapenemase-producing Enterobacterales ${ }^{5}$, is associated with risk of infection by these organisms. Metagenomic approaches are less biased than targeted approaches which capture specific organism/resistance phenotypes of interest, and obviate the need for culturing individual organisms. Resistance gene abundances and taxonomic distributions in metagenomes are increasingly mined for a range of applications in the study of AMR, including as 
correlates for national antibiotic exposures ${ }^{6,7}$ in the case of human gut metagenomes, or as an approach to monitoring global AMR in the case of sewage ${ }^{8}$. However, to our knowledge, no study to date has used taxonomic and resistome profiles of pooled metagenomes to directly estimate the AMR prevalence in clinical isolates within the same population, across a range of species and antimicrobial classes. This approach would enable intermittent, strategic sampling of a subset of individuals in a population to estimate the burden of AMR in clinical isolates, facilitating evidence-based development of empiric treatment guidelines without the need for isolate-based microbiological surveillance. Most samples taken to assay colonisation (e.g. faeces/rectal swabs, nasal/throat swabs) are relatively non-invasive and acceptable for individuals, and tolerated by particularly vulnerable groups, such as neonates.

The concept of a taxonomy-adjusted AMR metric or AMR resistance potential for a metagenome has been described previously $y^{6,9}$ as the average metagenome fraction encoding resistance genes for a particular antibiotic or antibiotic group, across all bacteria in a sample that can potentially carry such resistance genes, based on known taxonomic ranges for the resistance gene families. To model the benefit of such a metric in predicting resistance in clinical isolates within a population, we took pooled faecal samples from a sub-population of individuals $(>100)$ in three disparate geographic settings with varying AMR prevalence, namely Cambodia, Kenya and the United Kingdom (UK), and validated the model predictions using microbiological data from clinical isolates processed by laboratories in these locations over a seven-year period (2010-2017).

\section{Materials and Methods}

\section{Samples and Settings}

Faecal material stored in three existing biobanks was chosen for study; ethical approval for the broader use of these samples was in place. Samples comprised: (i) rectal swabs from children aged 159 months with and without malnutrition, taken on admission to Kilifi County Hospital in Kilifi, Kenya, from $1^{\text {st }}$ April to $30^{\text {th }}$ September 2016, and stored in Amies transport media $+1 \mathrm{ml}$ phosphate 
buffered saline at $-80^{\circ} \mathrm{C}$ ("Pharmacokinetics of Antimicrobials and Carriage of Antimicrobial Resistance amongst Hospitalised Children with Severe Acute Malnutrition (FLACSAM)' study $^{10}$ [KEMRI/SERU/CGMR- C/023/3161; OXTREC 47-15]); (ii) faecal samples taken from newborns on admission to Angkor Hospital for Children in Siem Reap, Cambodia, from $11^{\text {th }}$ September 2013 to $10^{\text {th }}$ September 2014 , and stored in tryptone soya broth $+10 \%$ glycerol at $-80^{\circ} \mathrm{C}^{11}($ OxTREC ref $1047-$ 13; this collection also included longitudinal samples taken from a subset of newborns during their inpatient stay for another study); and (iii), rectal swabs (Eswab, Copan diagnostics, Murrieta, CA, USA); $1 \mathrm{ml}$ Amies transport media) from individuals aged $\geq 18$ years attending pre-admission clinics or on admission to Guy's and St Thomas' NHS Foundation Trust, London, UK, between February and May 2015, and stored at $-80^{\circ} \mathrm{C}^{12-14}$ ( [REC: $\left.\left.14 / \mathrm{LO} / 2085\right]\right)$. Rectal swabs and faecal samples have both been used as approaches for surveying intestinal microbiota ${ }^{15,16}$, and are thought to give similar results $^{17}$.

For each study site, metadata associated with microbiology tests performed on blood and cerebrospinal fluid samples (as most robustly representative of true causative pathogens) collected within 0-72 hours of admission from 01/Jan/2010-31/May/2017 were collated. Each site has a microbiology laboratory participating in external quality assurance schemes (e.g. UK National External Quality Assessment Service, NEQAS) and is additionally accredited to UK ISO15189 (London laboratory) or WHO Good Clinical Laboratory Practice standards (Kilifi laboratory). Catchment areas served by each laboratory vary: For Cambodia about two-thirds of the patients come from within Siem Reap province ${ }^{18,19}$; in Kenya the population served is mostly rural, within the coastal Kilifi District ${ }^{20}$; and in London the laboratory largely serves a South London community of approximately 0.5 million people and also regularly provides services to international patients and patients from other sites in the $\mathrm{UK}^{21}$. Collated metadata included bacterial species identification results, available antibiotic susceptibility testing (AST) results, specimen type and basic patient details to validate aggregate-level stratification by age. Samples were processed using standard operating procedures in accordance with accredited guidelines. In the UK, the VITEK system (bioMérieux, Marcy-l'Etoile, France) was used for AST and performed according to the British Society for 
Antimicrobial Chemotherapy standards ${ }^{22}$ (BSAC). In Cambodia and Kenya, AST was performed using a standardised disk diffusion method following the Clinical and Laboratory Standards Institute (CLSI) guidelines ${ }^{23}$. Where accurate AST results could not be achieved by simple disk diffusion, minimum inhibitory concentrations (MICs) were determined by Etest in both settings. The infection metadata was collated for infants $<90$ days of age in Cambodia, $\leq 60$ months of age in Kenya and $\geq$ 18 years of age in the UK.

\section{DNA Extraction}

133 Samples from Cambodia and Kenya were shipped to the Nuffield Department of Medicine (University of Oxford, UK) for extraction; extractions for London samples took place at the Centre for Clinical Infection and Diagnostics Research (CIDR-King's College London). DNA was extracted from each sample using the MoBio PowerSoil@ DNA isolation kit (Qiagen, Hilden, Germany), as per the manufacturer's instructions with optimisation steps to achieve sufficient DNA yields for sequencing (ideally $\geq 300 \mathrm{ng} \mathrm{DNA} / 34 \mathrm{ul}$, with a view to obtaining $\geq 20 \mathrm{Gbp}$ (Giga base pairs) of data per sample). See Supplementary Methods $1 \& 2$. Known copy numbers of internal standards consisting of Thermus thermophilus HB8 genomic DNA ${ }^{24}$ (not normally present in faecal samples) were added to each sample prior to the addition of Solution C1 (i.e. 8.75 ul per sample [1ng/ul of Thermus DNA]).

The presence of T. thermophilus was ascertained following sequencing by mapping reads to the Thermus reference genome.

\section{Sample Pooling}

DNA extracts were stored at $-20^{\circ} \mathrm{C}$ and then pooled and sequenced at the Wellcome Trust Centre for

Human Genetics, Oxford, UK. For each study site, we created a "population pool", which consisted of the pooling of equimolar concentrations of all extracts from that setting with $\geq \operatorname{lng}$ DNA/ $\mu$ l. To validate our pooling approach, we also created one smaller pool in each setting, a so-called "30sample pool", which consisted of equimolar concentrations of 30 randomly selected extracts with $\geq 300$ ng DNA $/ 34 \mu$ l. An aliquot from each extract included in 30 -sample pools was in turn sequenced individually for the validation study (i.e. sequenced extracts from 90 individuals in total). An aliquot 
from all extracts sequenced individually and included in the 30-sample pools was also included in population pools.

\section{Metagenomic Sequencing}

Sequencing of all samples (pools and individual extracts) was performed using the HiSeq 4000 Illumina platform, generating $150 \mathrm{bp}$ paired-end reads (i.e. 96 metagenomes [n=90 individual metagenomes, $n=3$ 30-sample pools, $n=3$ population pools]). 500ng of DNA from each sample was used for library preparation. Libraries were constructed using the NEBNext Ultra DNA Sample Prep Master Mix Kit (NEB) with minor modifications and a custom automated protocol on a Biomek FX $(\text { Beckman })^{25}$. At the time of sequencing, the HiSeq 4000 produced on average $72-90$ Gbp of data per lane. We sequenced four individual extracts per lane to obtain on average 18-22.5 Gbp of data per sample. For the pooled samples, we sequenced one 30-sample-pool plus one population-pool per lane to obtain on average 36-45 Gbp of data per pool. Metagenomic data was obtained once for each distinct sample or pool; there were no technical replicates due to the expense of high-throughput sequencing.

\section{Sequence Data Processing}

We determined the taxonomic abundance of bacterial species and resistance genes at individual and pooled sample levels using a recently developed bioinformatics pipeline ${ }^{26}$. This pipeline incorporated established approaches to taxonomic profiling, and an adapted approach to quantify resistance gene markers present in a metagenome (for details of the method, see ${ }^{26}$ ). Briefly, the sequenced paired-end reads were quality-filtered based on PHRED scores ( $\geq$ Q25 and $\geq 50 \mathrm{bp}$ ), and adapters removed using TrimGalore ${ }^{27}$. For profiling the abundance of bacterial species, the quality-filtered sequences were classified with Kraken $2^{28}$ (v.2.0.8-beta) against bacteria, plasmid, viral and human genome sequences recovered (12 July 2019) from NCBI. With the taxonomic classification from Kraken2 and information about species specific versus non-specific genetic regions we estimated true abundance at the species level using Bracken ${ }^{29}$ (v.2.5.0), which was subsequently used for deriving total aggregate counts of bacterial taxa. For profiling resistance genes, the quality-filtered sequences were mapped 

(v.37.72) at $100 \%$ sequence identity. The number of sequences that mapped to each resistance gene were subsequently corrected to remove resistance gene length bias. This was done using four metrics, namely (1) specific read count (number of sequences that map exclusively to the resistance gene); (2) specific lateral coverage (proportion of the resistance gene covered by sequences mapping exclusively to the gene); (3) resistance gene length; and (4) and average read length (average length of reads that mapped to the resistance gene), and by the following formula: corrected gene count $(\mathrm{CGC})=($ specific read count $\mathrm{x}$ average read length) / (resistance gene length $\mathrm{x}$ specific lateral coverage).

The CARD database attempts to classify each resistance gene variant by its association with AMR. To be included in CARD, an AMR determinant must be described in a peer-reviewed scientific publication, have its DNA sequence available in GenBank, and include clear experimental evidence of elevated MIC over controls ${ }^{31}$. We used these data to map and aggregate counts of resistance genes/variants associated with resistance to a specific antibiotic. In the process, we ranked the resistance genes/variants into two categories, reflecting to some extent the public health risks posed ${ }^{33}$, and thereby creating two sets of antibiotic resistance gene metrics. The first (AMR ${ }_{\mathrm{DEF}}$; Supplementary

Data 1), included only AMR determinants with the "Confers_Resistance_to_Antibiotic" relationship ontology term, whereby the gene associated with demonstrably elevated MIC is known to confer or contribute to clinically relevant resistance to a specific antibiotic drug ${ }^{31}$. The second $\left(\mathrm{AMR}_{\mathrm{ALL}}\right.$; Supplementary Data 2), contained corrected counts of all resistance genes with clear experimental evidence of increasing the MIC, including those associated with clinically relevant resistance (as for $\left.\mathrm{AMR}_{\mathrm{DEF}}\right)$, plus those without the "Confers_Resistance_to_Antibiotic" relationship ontology term. For the purposes of this study we have used the term "resistance gene" to define any relevant genetic marker of resistance, including genes that confer resistance by mutation (but can have a susceptible wild type), and genes that confer resistance through presence/absence. 
We evaluated to what extent pooled resistome data was a non-biased representation of the individual resistomes making up the pool. Resistance gene abundances of the 30 -sample pools and individually sequenced samples were converted to relative abundances, such that gene abundances in each sample summed to one. Then, for each of the three different settings, individual samples were used to compute the empirical distribution of each gene by repeated random sampling of its relative gene abundance out of the individual samples (bootstrapping with $n=100,000$ repeats). We were then able to compare the pool abundance of each gene with its empirical distribution in the same setting (within-setting comparison) and in the other two settings (across-setting comparison). We computed the fraction of resistance genes for which the pool estimate was within $90 \%$ central quantile of the empirical distribution. The resulting metric was restricted between 0 (i.e. $0 \%$ of resistance genes in the pool were as expected given the individual resistomes) and 1 (i.e. $100 \%$ of resistance genes in the pool were as expected). Because bootstrapping of gene abundances relies on having a sufficient number of samples with non-zero abundance, we limited our analysis to genes present in $\geq 50 \%$ of all individual samples ( $\mathrm{n}=121$ genes). Given the central quantile choice above (i.e. $90 \%$ ), a value of $\sim 0.90$ would imply a non-biased representation of individual resistomes by the pooled resistome. For visualization, non-metric multidimensional scaling, an ordination-based method, was used to show pair-wise dissimilarities between resistomes from population pools, 30-sample-pools and individual sample means within and across settings. Individual sample means for each setting were, for each AMR gene, the sum of CGCs across all individually sequenced samples.

\section{Taxonomy Adjusted Resistance Potential Metrics}

We developed several candidate metrics of resistant infection risk, based on pool metagenomic data on resistance gene abundance and bacterial species composition, and evaluated their potential to accurately predict the likelihood of antibiotic resistant invasive infections in a population. We refer to these as 'taxonomy-adjusted resistance potential (RP)' metrics, which consisted of two parameters. The first parameter, $R_{C G C}$, was given through the sum of corrected gene counts (CGC) of variants associated with resistance to a given antibiotic, $j\left(R_{C G C j}\right)$ divided by the total CGC of all resistance genes in the pool. $R_{C G C, j}$ was calculated based on either variants with experimental evidence of 
increasing the $\mathrm{MIC}\left(\mathrm{AMR}_{\mathrm{ALL}}\right)$ or only variants known to confer clinically relevant resistance

237 (AMR $\mathrm{DEF})$. The second parameter, $R_{\text {Tax }}$, was given through the estimated abundance of a clinically relevant bacterial grouping (derived from Bracken estimates) divided by the total estimated abundance of bacterial taxa in the pool. The bacterial groupings tested were the Enterobacterales order, Enterobacteriaceae family, and the grouping of the four most common and clinically relevant bacterial genera/species within the Enterobacteriaceae family across sites (namely Escherichia coli, Klebsiella pneumoniae, Salmonella spp, Enterobacter spp).

\section{Bayesian Modelling}

With each taxonomy-adjusted RP, we fitted a Bayesian generalized linear model to the data and applied model comparison. This allowed us to assess the potential of the different metrics to predict observed antibiotic resistance amongst clinical invasive Enterobacterales isolates. We used deduplicated counts of isolates (unique bacterial species per antibiogram and patient-ID) for the analyses. We let $i$ denote the setting (Cambodia, Kenya or UK), and $j$ the antibiotic (see below for a list). We assumed that counts of resistant samples follow a binomial distribution. Our model then predicts the count of resistance $\left(r_{i, j}\right)$ among tested Enterobacterales isolates $\left(n_{i, j}\right)$ using a probability of resistance $\left(p_{i, j}\right)$, which is modelled as

The model intercept $(\alpha)$ is specific for each antibiotic $(j)$ but not setting $(i)$, representing a baseline propensity of resistance for any given antibiotic. Because resistance propensities can vary widely between different antibiotics. We assume independent baselines (fixed effects). The setting-specific information is $R_{T a x, i}$, which gives information about pathogen levels in setting $i$, as well as $R_{C G C, i, j}$, which carries information about resistance toward antibiotic $j$ in setting $i$. For $\beta_{1, \mathrm{j}}$ and $\beta_{2, \mathrm{j}}$, the predictive effects of $R_{C G C}$ and $R_{T a x}$, we assumed these to represent the clinical ecology of resistance 
genes so that they are specific to each antibiotic, $j$, but not to each setting, $i$. We further assumed that different antibiotics have different but related $\beta$-values (variable effects, specified below). We included only those antibiotics that had existing antibiotic susceptibility test (AST) data in at least two out of three settings (trimethoprim-sulfamethoxazole, nitrofurantoin, nalidixic acid, meropenem, imipenem, gentamicin, ciprofloxacin, chloramphenicol, cefuroxime, ceftriaxone, ceftazidime, cefpodoxime, cefoxitin, cefotaxime, ampicillin, amikacin); missing observations were excluded from the likelihood evaluation. We fitted the above model with $R_{C G C}$ being either $\mathrm{AMR}_{\mathrm{DEF}}$ or $\mathrm{AMR}_{\mathrm{ALL}}$ and with $R_{\operatorname{Tax}}$ being either of the three bacterial groupings discussed earlier, yielding a total of six separate model fits. Due to the limited number of infection isolates with AST results (especially in Cambodia), we chose standard weakly informative priors for the intercept and the effect parameters. In addition, we restricted the effect of gene abundance to be positive, reflecting our view that only a positive association of resistance genes and clinical resistance is biologically reasonable. We therefore chose

$$
\begin{gathered}
\beta_{1, j} \sim N^{+}\left(\mu_{\beta, 1}, \sigma_{\beta, 1}\right) \\
\beta_{2, j} \sim N\left(\mu_{\beta, 2}, \sigma_{\beta, 2}\right) \\
\alpha_{j} \sim N(0,1) \\
\mu_{\beta, 1} \sim N^{+}(0,1) \\
\mu_{\beta, 2} \sim N(0,1) \\
\sigma_{\beta, 1} \sim N^{+}(0,1) \\
\sigma_{\beta, 2} \sim N^{+}(0,1)
\end{gathered}
$$

where $N$ denotes a normal distribution and $N^{+}$denotes a half-normal distribution covering only positive values. Each model was fit using Stan $\operatorname{software}^{34}$ (v2.19.1), with which we sampled 50,000 samples after a burn-in period of 5,000 samples using four independent chains.

The best taxonomy-adjusted RP metric was selected using Bayesian leave-one-out cross validation ${ }^{35}$ which estimates a model's pointwise out of sample prediction accuracy. The prediction accuracies are 
then used to directly compare all models using stacking weights ${ }^{36}$. In brief, models with smaller crossvalidation errors (e.g. smaller prediction errors), get more weight relative to other models in the model comparison. We also included in the comparison two models with $R_{C G C}$ (either $\mathrm{AMR}_{\mathrm{DEF}}$ or $\left.\mathrm{AMR}_{\mathrm{ALL}}\right)$, following Equation (1), but without $R_{\text {Tax }}$. Finally, the overall value of using any taxonomy-adjusted RP metric for predicting clinical resistance was assessed by including in the model comparison a baseline model without predictors. The prediction accuracy of taxonomy-adjusted RP was also assessed visually by comparing the best model's predictions of sample counts of resistance (and their 95\% credible intervals $[\mathrm{CI}]$ ) against the observed counts (Figure 5). For settings and antibiotics where zero samples were tested, we imputed the sample size by computing the rounded mean of the sample sizes of the other two settings. Model comparisons and all further data analyses were performed in R3.6.1 statistical software ${ }^{37}$. The dataset used for the Bayesian modelling is given in Supplementary Data 3.

\section{Results}

The study included 210 admission samples from Kenya, 200 from the UK and 153 from Cambodia ( $\mathrm{n}=154-1$ rejected sample), totalling 563 samples for metagenomic analysis (Fig 1). In addition, 76 follow-up samples were taken from 37/154 newborns in Cambodia during their inpatient stay or upon hospital discharge for a separate project; these were processed alongside the study samples (Fig 1). We only considered DNA extracts with yields $\geq 1 \mathrm{ng} / \mathrm{ul}$ (79-89\% of samples; Fig 1), and 19 DNA extracts from the separate longitudinal study were included in the Cambodia population pool due to processing error. In total, population pools in Kenya, the UK and Cambodia, comprised 177, 157, and 156 pooled sample extracts. Thirty high DNA-yield samples $(\geq 9 \mathrm{ng} / \mathrm{ul})$ from each setting were used for the validation study, as well as being included in the population pools. To prevent bias, potential associations between high-yield samples and population traits were ruled out in advance. The total Gbp of data obtained per population pool were 51.6 (Kenya), 55.1 (UK) and 52.6 (Cambodia). The median Gbp obtained for individually sequenced samples were 24.2 (Kenya), 22.1 (Cambodia), 22.4 (UK). 


\section{Fig 1. Sample Processing Workflow}

319

320

We identified 863 different antimicrobial resistance genes across any sample or pool (Cambodia: 684; Kenya $=527 ; \mathrm{UK}=520)$, which were proven to increase the MIC for 163 antimicrobials $\left(\mathrm{AMR}_{\mathrm{ALL}}\right)$ and known to confer clinically relevant resistance for 113 antimicrobials (AMR $\mathrm{DEF})$. The number of resistance genes identified in population pools was largest in Cambodia $(n=490)$, followed by the UK $(n=389)$ and Kenya $(n=386)$. The median number of resistance gene types identified per individual sample was also higher in Cambodia (median=162; IQR=126-187 [Min-Max =33-231]), followed by in Kenya (median=143; IQR= 127-205 [Min-Max =97-256]) and UK (median=134; IQR=126-148; $[$ Min-Max =61-217]).

A summary of the Enterobacterales taxa identified from population pools and invasive infections in each setting is given in Fig 2. Enterobacterales were the main bacterial taxa identified from population pools in the UK (75.7\%) and Cambodia (69.7\%) but not in Kenya (32.4\%) (Fig 2A). Within the Enterobacterales, $>95 \%$ of the bacteria were from the Enterobacteriaceae family in all settings (UK: 96.3\%; Cambodia: 99.4\%; Kenya: 99.1\%). The predominant species within the Enterobacterales order in population pools were E. coli and K. pneumoniae, followed by Enterobacter spp. (Fig 2E). These species and genera combined accounted for $92.4 \%$ of all Enterobacterales taxa in Kenya, $88.5 \%$ in the UK and $88.1 \%$ in Cambodia. The abundance of E. coli, was $>20$-fold higher than that of $K$. pneumoniae in population pools from the UK (E. coli: $63.2 \%$; K. pneumoniae: $2.2 \%)$ and Kenya (E. coli: $28.4 \%$; K. pneumoniae: $1.3 \%$ ). In contrast both species had similar abundance in the Cambodia population pool (E. coli: 30\%; K. pneumoniae: 26.9\%). Enterobacter spp. abundance was also higher in Cambodia (4.5\%) compared to the UK $(1.6 \%)$ or Kenya $(0.2 \%)$. The remaining Enterobacterales comprised other genera, each being $<2 \%$ of the total bacterial taxa in the three settings (Fig $2 \mathrm{G}$ ). Infections by Enterobacterales accounted for approximately a third of all blood and cerebrospinal fluid infections in the three settings (Kenya: 36.8\%; Cambodia: 33.0\%; UK: 28.2\%) (Fig 2B). Similar to the findings from population pools, most of these Enterobacterales infections involved the 
346 Enterobacteriaceae family (UK: 91.2\%, Cambodia: 89.2\%; Kenya: 91.8\%; Fig 2B). Likewise, the

347 predominant Enterobacterales species in all settings were E. coli and K. pneumoniae, with the

348 proportion of $E$. coli infections being at least double that of $K$. pneumoniae in the UK (E. coli: $16.1 \%$;

349 K. pneumoniae: 4.8\%) and Kenya (E. coli: 13.8\%; K. pneumoniae: 5.9\%), but not in Cambodia (E.

350 coli: $13.7 \%$; K. pneumoniae: 11.7\%) (Fig 2F). Enterobacter spp. was the next most common

351 Enterobacterales genus in all settings (Cambodia: 3.1\%; Kenya: 2.7\%; UK: 2.2\%), but the remaining

352 Enterobacterales species and genera accounted for $<2 \%$ of the total invasive infections by any

353 bacterial order each in all three settings (Fig 2H). A notable exception was Salmonella spp., which

354 accounted for $9.9 \%$ of the total infections in Kenya (therefore also included in Fig $2 \mathrm{~F}$ and the

355 equivalent plot for population pools [Fig 2E]). Details of all invasive infections by bacteria other than

356 the Enterobacterales are given in Supplementary Fig 1.

357 


\section{Fig 2. Bacterial (Enterobacterales) taxa in population pools and in blood and cerebrospinal fluid infections from Cambodia, Kenya and UK.}

Panels for population pools (A, C, E, G) show, for each setting, the abundances of Enterobacterales taxa divided by the total abundance of bacterial taxa in a pool. Abundances are derived from Bracken estimates. Panels for invasive infection data $(B, D, F, H)$, show percentages of Enterobacterales infection isolates out of all bacterial infection isolates with speciation results identified from blood and cerebrospinal fluid samples in target age groups, in each setting, from 2010-2017 (Cambodia [ $n=197]$; Kenya [ $n=910]$; UK [ $n=3356]$ ).

The highest relative abundances of resistance genes observed in each setting were for genes associated with resistance to aminoglycosides, amphenicols, fluoroquinolones, tetracyclines and macrolides $(48.1 \%, 45.8 \%$ and $43.6 \%$ of the total counts in Cambodia, Kenya and the UK respectively) (Fig 3A, left-hand panel). Relative abundance of resistance genes associated with these five broad antibiotic classes differed between settings. For example, the relative abundance of resistance genes for aminoglycosides in Cambodia (18.4\%) was almost double that in Kenya (10.8\%) or UK (10.9\%). The next highest relative abundance was of genes conferring resistance to penicillins (Cambodia: 4.1\%; Kenya: 4.7\%; UK: 5.0\%) and cephalosporins (Cambodia: 2.6\%; Kenya: 2.3\%; UK: $2.2 \%$ ). Resistance gene counts for other antibiotic classes were $<2 \%$ of the total gene counts in all settings, including to carbapenems (Kenya [0.5\%], Cambodia and UK [0.4\%]). For single antibiotics or antibiotic sub-classes (e.g. $1^{\text {st }}$ generation cephalosporins), the highest relative abundances were observed for erythromycin (Cambodia: 3.9\%; Kenya: 4.2\%; UK: 4.4\%) and chloramphenicol (Cambodia: 3.6\%; Kenya: 3.5\%; UK: 4.2\%) in all settings (Fig 3A, right-hand panel). That for resistance genes to antibiotics other than those listed was $76 \%$ (Cambodia), $76.5 \%$ (Kenya) and $76.1 \%$ (UK) (data not shown). The relative abundance of resistance genes for all other single antibiotics/antibiotic sub-classes was $<2 \%$ in all settings, except for tigecycline (Cambodia: $2.8 \%$; Kenya and UK: 2.2\%) and clindamycin (Kenya: 2.1\%; UK: 2.6\%). Resistance prevalence in Enterobacterales isolates causing blood and cerebrospinal fluid infections is displayed in Fig.3B (right-hand panel) for the 16 antibiotics with antibiotic susceptibility test data in $\geq 2$ settings. For comparison, this is shown alongside the relative abundance of resistance genes for the same antibiotics in population pools (Fig.3B, left-hand panel). 
Fig 3. Relative abundance of resistance gene counts in population pools and percentage of resistant Enterobacterales blood and cerebrospinal fluid infections in Cambodia, Kenya and UK.

Panels in Fig 3A show, for each setting, corrected resistance gene counts (CGCs) for a given antibiotic, antibiotic class, or sub-class, divided by the total corrected AMR gene counts identified in the population pool. Relative abundances were calculated using AMR $\mathrm{ALL}_{\mathrm{L}}$, which considers corrected counts of genes and variants (CGC) increasing the MIC or conferring clinically relevant resistance for a given antibiotic. Panels in Fig 3B show, for each setting, the observed percentage of Enterobacterales resistant infections for 16 antibiotics with AST data in $\geq 2$ settings (right-hand side), and the relative abundance of CGCs for the same antibiotics in population pools, based on AMRALL (left-hand side). Percentages are shown with 95\% exact binomial confidence intervals in both panels.

Pair-wise dissimilarities in resistomes from population pools, 30 -sample-pools and individual sample means (i.e. sum of CGC for the resistance gene types across all individually sequenced samples) were calculated both within and across settings (Fig 4A and 4B), considering either the absolute CGC values for each resistance gene type or their relative abundance based on the CGC values. Population pools, 30-sample pools and individual sample means were less dissimilar and hence more closely related within settings than across settings. In addition, within each setting, individual sample means were more often less dissimilar to 30-sample pools than to population pools. In Cambodia 362 AMR genes were identified in the 30 -sample pool compared to 616 across all 30 individual samples. The 30-sample pool in Kenya comprised 339 genes compared to 499 across all individual samples. Finally, in the UK 318 AMR genes were identified from the 30-sample pool compared to 422 across all individual samples. However, when comparing individual samples and pools from the same setting quantitatively, the average fraction of resistance genes for which the 30-sample pool estimate was within the central interval of the empirical distribution inferred from individually sequenced samples was 97\% (Kenya: 98\%; Cambodia: 97\%; UK: 95\%). In contrast, the average fraction was $86 \%$ across comparisons between different settings (min-max: 80-92\%). All 30-sample pool resistomes therefore had substantially higher similarity to individual resistomes from the same setting relative to the comparison with other settings. 
bioRxiv preprint doi: https://doi.org/10.1101/2020.02.10.941930; this version posted February $11,2020$. The copyright holder for this preprint (which was not certified by peer review) is the author/funder, who has granted bioRxiv a license to display the preprint in perpetuity. It is made available under aCC-BY-NC-ND 4.0 International license. error of 33.

\section{Fig 4. Pair-wise dissimilarities in the resistome of population pools, 30-sample-pools and individual sample means within and across settings}

Using non-metric multidimensional scaling (NMDS) ordination-based method, Fig 4 shows pair-wise dissimilarities of resistance gene counts from population pools (PP), 30-sample-pools (30S) and individual sample means (SI) within and between settings, following mapping of sequences from individual and pool metagenomes against CARD and a correction to remove resistance gene length bias from counts. Dissimilarities are shown for the absolute corrected resistance gene counts (CGC; left hand-side) and the relative abundance of resistance genes (right hand-side). Relative abundances for genes in pools were calculated by dividing the CGC for each gene by the total CGC of all resistance genes in the pool. Individual sample means were, for each resistance gene, the sum of CGC across all individually sequenced samples. This, divided by the total CGC of all resistance genes across all individually sequenced samples, was the relative abundance of each resistance gene based on individual sample means.

The best taxonomy-adjusted RP metric - resulting in the highest point-wise out of sample prediction accuracy and the greatest relative model weight - used the taxonomic parameter $R_{\operatorname{tax}}$ measuring Escherichia coli, Klebsiella pneumoniae, Salmonella spp. and Enterobacter spp., and the abundance

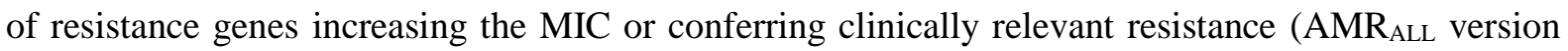
of the $R_{C G C}$ metric). This $\mathrm{AMR}_{\mathrm{ALL}}$ model outperformed the other models, including a baseline model without any metagenomics information, plus those models without taxonomic $\left(R_{t a x}\right)$ information (Bayesian model averaging weights: Baseline [no $R_{C G C}$ and no $R_{t a x}$ ] $=0 ; R_{C G C}$ only [No $R_{t a x}$ ] $=0$; Best model $=0.47]$. Supplementary Data 4).

Model predictions were made for 16 antibiotics, which were those that had antibiotic susceptibility test (AST) data for Enterobacterales isolates causing infection in at least two of the three settings (Supplementary Data 5). Our best model accurately predicted the number of resistant infections in the target populations for $100 \%$ of antibiotics with AST data in Kenya (12/12) and UK (14/14). In Cambodia, the model accurately predicted the counts of resistant infections for $75 \%$ of antibiotics (9/12). Compared to this, the baseline model did not correctly predict $50 \%$ of antibiotics across the three settings (19/38). We computed the mean-squared errors of the mean model predictions relative to the observations. The baseline model had an error of 468, whilst the final model (Fig 5) had an 

resistance to antibiotics with antibiotic susceptibility test (AST) results in $\geq 2$ settings.

Horizontal bars represent $95 \%$ highest density posterior interval and vertical lines represent means of the predicted resistant sample counts based on the model using metagenomic data from population pools. Coloured bars (yellow: Cambodia; blue: Kenya; brown: UK) are shown where clinical data on resistance (i.e. AST) was available and grey bars where it was not. For grey bars the sample size was imputed. Red circles show the number of blood and cerebrospinal fluid Enterobacterales infections that were found to be resistant to the antibiotic listed in the $y$-axis. The number of isolates with AST results are also given in the $y$-axis. The red circle is missing where no AST results were available. In cases where there is minimal uncertainty in the model estimate, the red circle may overshadow the $95 \%$ credible interval bars (e.g. meropenem [Cambodia]; cefuroxime [Kenya]). "Trimethoprim." is short for trimethoprim-sulfamethoxazole; "Cloramph" is short for chloramphenicol. NT = no AST data available.

Bayesian model predictions expressed as percentages are shown in Supplementary Fig 2 for antibiotics where AST results were available from > 100 invasive infection isolates. Above this threshold, predicted percentage resistance was accurate for $100 \%$ of antibiotics (14/14 with >100 tested isolates).

\section{Discussion}

In this study we have demonstrated the feasibility of a novel, pragmatic approach to surveillance of bacterial antimicrobial resistance of relevance to human infection, with a focus on Enterobacterales as one of the major bacterial resistance threats ${ }^{38,39}$. Our results show that metagenomic analysis of pooled faecal material (pooled at equimolar concentrations) is effective at predicting invasive infections caused by Enterobacterales resistant to in-use antibiotics in a population, across a range of different age groups and geographic settings. Our approach would enable intermittent, acceptable and relatively non-invasive sampling of a small number of individuals within a population (e.g. 100-200), with the advantage that a single centralised infrastructure (either in-country or internationally) could undertake the metagenomic sequencing and analysis. This can be done independently of development of a network of classical microbiological laboratories in multiple settings, which can be resource-intensive in terms of capital and running costs, and is not feasible in the short-term, especially in LMICs, which frequently have the highest AMR burden. 
Based solely on pool size and sequencing depth $(50-55 \mathrm{Gbp} / \mathrm{pool})$, we developed predictive metrics (RP) without the need for costly and labour-intensive multiplexing of samples (i.e. individually identifying samples in the pool by means of barcoded sequences) or selective sequencing approaches based on enrichment for predefined panels of resistance genes. Unlike other AMR gene profiling approaches our bioinformatics pipeline (ResPipe) incorporates the capacity to identify both specific AMR gene variants (e.g. such as bla $a_{\mathrm{CTX-M-33}}$ versus $b l a_{\mathrm{CTX- \textrm {M } - 6 3}}$ ), as well as being able to aggregate by gene family. This is especially important for the prediction of phenotypes, as genes that differ by only single nucleotides/amino acids can have distinct phenotypic spectra. Pooled metagenomes/resistomes were also found to be an accurate, non-biased representation of the individual sample metagenomes/resistomes. Population pools comprising rectal swabs with as little as $\geq 1 \mathrm{ng} / \mathrm{ul}$ DNA/sample were found to be sufficient to derive RP metrics with predictive value; this is useful in terms of optimizing the sample processing workflows. Finally, in producing relatively deeply sequenced (50-55Gbp/metagenome) and complete (i.e. not restricted to $16 \mathrm{~S}$ ) metagenomes on 90 individuals, we have also made a significant contribution to the human microbiomics data repository, freely available for other researchers to use for study.

The limitations of our approach were most obvious for the neonatal group from Cambodia, where predicted resistance matched the observed resistance in invasive isolates for $75 \%$ of antibiotics compared to $100 \%$ of antibiotics in Kenya and the UK. One explanation for this might be that the population pool for this group was found to have included 19 longitudinal samples (12\% of all samples in the pool) collected from individuals during their hospital inpatient stay, potentially biasing the metagenomics profile of population pools and infection metadata designed to reflect community (i.e. non-hospital) profiles. Rapid changes in the neonatal resistome occur following exposure to the

510 hospital environment ${ }^{40}$. Analyses of neonatal metagenomes have shown that these are predisposed to 511 rapid flux, and in hospital typically reflect the environmental hospital "microbiome"41. Cambodia was 512 also the only setting where the age group considered for metagenomics analysis (i.e. neonates), did 
bioRxiv preprint doi: https://doi.org/10.1101/2020.02.10.941930; this version posted February $11,2020$. The copyright holder for this preprint (which was not certified by peer review) is the author/funder, who has granted bioRxiv a license to display the preprint in perpetuity. It is made available under aCC-BY-NC-ND 4.0 International license.

514

515

516

517

518

519

520

521

522

523

524

525

526

527

528

529

530

531

532

533

534

535

536

537

538

539

age), which may also have influenced the accuracy of our predictive approach. Our analysis was also limited by the scarce antibiotic susceptibility test (AST) results available for invasive infection isolates, particularly in Cambodia, where the maximum number of isolates with AST results for any given antibiotic was 65, compared to 324 in Kenya and 912 in UK. The smaller number of isolates from Cambodia meant that the model fit contained less information to accurately predict resistance in this setting. Moreover, AST results were only available for a limited number of antibiotics across all three settings, and ideally AST approaches used for comparison would have been standardised across the settings. Finally, our analyses are heavily dependent on the robustness of the reference gene database, and the accuracy of genotypic-phenotypic correlations catalogued therein. In general, however, we would expect this knowledge base to become increasingly robust, thus strengthening our predictions. This may explain why in this study, a model that considers all gene variants with experimental evidence of increasing the minimum inhibitory concentration (MIC), outperformed a model considering only genes known to confer clinically relevant resistance.

Further studies to validate our promising proof-of-principle observations in additional settings across age categories, especially the neonatal group, are warranted. There is potential to extend the approach to consider other priority bacterial groups and different colonisation samples. For example, pools could be extended to include samples from nasopharyngeal sites, where other potential pathogens predominate (e.g. Streptococcus spp., Staphylococcus spp.). To develop the most rapid, convenient, simple and inexpensive method possible, future studies should also consider further simplifications to the method such as whether the same accurate predictions can be generated by pooling all samples prior to DNA extraction and then performing the extraction only once. Further work should also test the resolution of the approach to characterise and track local/sub-national variation in AMR prevalence, or in community versus healthcare-associated contexts. A mathematical framework for minimum-cost implementation of pooled-sample metagenomics-based surveys to quantify the burden of resistance in new settings without prior microbiology or AST data would also be of benefit, and 
could be greatly informed by the data we have generated, which can contribute to simulation work addressing pools sizes, pool numbers per region, and sequencing depth.

We conclude that surveillance based on population colonisation metagenomics and taxonomyadjusted AMR metrics presented here are in principle a valuable public health opportunity, and may represent an alternative or bridging measure to the implementation of local and regional laboratorybased infrastructures focussed on culturing isolates from clinical specimens, especially in resourcelimited settings. This novel approach could be used to overcome the current paucity of quality AMR surveillance data and inform setting-tailored rationalization of/or access to antibiotics, contextappropriate treatment guidelines, organized measures to prevent AMR and ultimately public-health decision in conjunction with relevant stakeholders, especially in LMICs.

\section{Ethics Declarations}

553 This research was conducted with approval from the Oxford Tropical Research Ethics Committee (OxTREC Reference: 5126-16) following local ethics clearance for inclusion of Cambodia and Kenya sample collections, and approval of a substantial amendment to 14/LO/2085 by the National Research Ethics Service (NRES London - Camberwell St Giles), for inclusion of the London sample collection in this study.

The authors declare no competing interests.

\section{Data and Code Availability}

562 The raw sequence data reported in this study have been deposited in the European Nucleotide Archive under accession number PRJEB34871. The code to extract CARD data, that required to generate the final datasets and analyses, plus any required input files, are available from the ResPipe GitLab repository (https://gitlab.com/hsgweon/ResPipe); ResPipe output data can be found at the ResPipe Gitlab subdirectory (https://gitlab.com/hsgweon/ResPipe/tree/master/data). 


\section{Author Contributions}

569 This work was first conceived by O.T.A., with support from N.S. and B.S.C.; O.T.A, N.S., B.S.C., 570 R.N. and H.S.G. designed the study. K.C., J.W., O.T.A. and N.S. developed and validated modified 571 DNA extraction protocols for this study. K.C., J.W. and R.B. conducted or facilitated most of the presample-pooling laboratory work. S.L. designed the methods and provided technical guidance for sample pooling and sequencing and conducted the sequencing work. J.A.B., J.D.E., P.T. and R.B. facilitated the collation and transfer of samples and data from participant settings. They also provided technical support for clinical and microbiology study procedures and for the development of contextappropriate standard operating procedures. N.S., A.S.W., T.E.P., D.W.C. and B.S.C. provided support and guidance for all technical aspects of the study (including for bioinformatics and data analyses) and contributed to the revision of study outputs. T.N. contributed to the mining, standardisation and analysis of infection metadata from each setting. H.S.G. conducted the bioinformatics work, designed the methods for corrected gene counts and extracted the data from CARD. J.S. provided the computing support for the study. O.T.A. conducted mining, linkage and visualisation of study data. R.N. conducted the validation and Bayesian analyses and B.S.C. contributed to revision of these methods. O.T.A, N.S., R.N. and H.S.G. produced the first manuscript draft. All authors contributed significantly to the iterative review of the draft.

\section{Acknowledgements}

We are grateful to Professor Mike English (Centre for Tropical Medicine and Global Health, University of Oxford, UK), for facilitating the initiation of this work by bringing together some of the groups involved in the research. We are also grateful to Dr Alessandra Natale, for supporting the laboratory work for this study at the CIDR (Centre for Clinical Infection and Diagnostics Research, Oxford. 
595

596

597

598

599

600

601

602

603

604

605

606

607

608

609

610

611

612

613

614

615

616

617

618

619

620

621

622

623

624

625

626

627

628

629

630

631

632

633

634

635

636

637

\section{References}

1. World Health Organisation. Global action plan on Antimicrobial Resistance. http://www.who.int/antimicrobial-resistance/global-action-plan/en/ (2016).

2. Collignon, P., Beggs, J. J., Walsh, T. R., Gandra, S. \& Laxminarayan, R. Anthropological and socioeconomic factors contributing to global antimicrobial resistance: a univariate and multivariable analysis. Lancet Planet. Heal. 2, e398-e405 (2018).

3. World Health Organisation. Global Antimicrobial Resistance Surveillance System (GLASS). http://www.who.int/drugresistance/en/ (2015).

4. Wertheim, H. F. L. et al. Risk and outcome of nosocomial Staphylococcus aureus bacteraemia in nasal carriers versus non-carriers. Lancet 364, 703-705 (2004).

5. Tischendorf, J., de Avila, R. A. \& Safdar, N. Risk of infection following colonization with carbapenem-resistant Enterobactericeae: A systematic review. Am. J. Infect. Control 44, 539543 (2016).

6. Forslund, K. et al. Country-specific antibiotic use practices impact the human gut resistome. Genome Res. 23, 1163-9 (2013).

7. Korpela, K. et al. Intestinal microbiome is related to lifetime antibiotic use in Finnish preschool children. Nat. Commun. 7, 10410 (2016).

8. Hendriksen, R. S. et al. Global monitoring of antimicrobial resistance based on metagenomics analyses of urban sewage. Nat. Commun. 10, (2019).

9. Forslund, K., Sunagawa, S., Coelho, L. P. \& Bork, P. Metagenomic insights into the human gut resistome and the forces that shape it. Bioessays 36, 316-29 (2014).

10. Standing, J. F. et al. Dosing of Ceftriaxone and Metronidazole for Children With Severe Acute Malnutrition. Clin. Pharmacol. Ther. 104, 1165-1174 (2018).

11. Turner, P. et al. High prevalence of antimicrobial-resistant gram-negative colonization in hospitalized cambodian infants. Pediatr. Infect. Dis. J. 35, 856-861 (2016).

12. Otter, J. A. et al. Universal hospital admission screening for carbapenemase-producing organisms in a low-prevalence setting. J. Antimicrob. Chemother. 71, (2016).

13. Dyakova, E. et al. Efficacy and acceptability of rectal and perineal sampling for identifying gastrointestinal colonisation with ESBL-Enterobacteriaceae. Clinical Microbiology and Infection http://www.sciencedirect.com/science/article/pii/S1198743X17301167 (2017) doi:10.1016/j.cmi.2017.02.019.

14. Otter, J. A. et al. Individual- and community-level risk factors for ESBL Enterobacteriaceae colonization identified by universal admission screening in London. Clin. Microbiol. Infect. $\mathbf{0}$, (2019).

15. Budding, A. E. et al. Rectal swabs for analysis of the intestinal microbiota. PLoS One 9, e101344 (2014).

16. Baumann-Dudenhoeffer, A. M., D’Souza, A. W., Tarr, P. I., Warner, B. B. \& Dantas, G. Infant diet and maternal gestational weight gain predict early metabolic maturation of gut microbiomes. Nature Medicine vol. 24 1822-1829 (2018).

17. Bassis, C. M. et al. Comparison of stool versus rectal swab samples and storage conditions on bacterial community profiles. BMC Microbiol. 17, (2017).

18. Chheng, K. et al. A Prospective Study of the Causes of Febrile Illness Requiring Hospitalization in Children in Cambodia. PLoS One 8, (2013). 
19. Fox-Lewis, A. et al. Antimicrobial resistance in invasive bacterial infections in hospitalized children, Cambodia, 2007-2016. Emerg. Infect. Dis. 24, 841-851 (2018).

20. Scott, J. A. G. et al. Profile: The Kilifi health and demographic surveillance system (KHDSS). Int. J. Epidemiol. 41, 650-657 (2012).

21. Tosas Auguet, O. et al. Frequent Undetected Ward-Based Methicillin-Resistant Staphylococcus aureus Transmission Linked to Patient Sharing Between Hospitals. Clin. Infect. Dis. 66, 840-848 (2018).

22. British Society for Antimicrobial Chemotherapy. http://www.bsac.org.uk/.

23. Clinical and Laboratory Standards Institute (CLSI). Performance Standards for Antimicrobial Susceptibility Testing. CLSI document M100-S27. (Wayne, PA: Clinical and Laboratory Standards Institute, 2017).

24. Satinsky, B. M., Gifford, S. M., Crump, B. C. \& Moran, M. A. Chapter Twelve - Use of Internal Standards for Quantitative Metatranscriptome and Metagenome Analysis. in Methods in Enzymology vol. 531 237-250 (2013).

25. Lamble, S. et al. Improved workflows for high throughput library preparation using the transposome-based nextera system. BMC Biotechnol. 13, (2013).

26. Gweon, H. S. et al. The impact of sequencing depth on the inferred taxonomic composition and AMR gene content of metagenomic samples. Environ. Microbiome 14, 7 (2019).

27. Babraham Bioinformatics - Trim Galore! http://www.bioinformatics.babraham.ac.uk/projects/trim_galore/.

28. Wood, D. E. et al. Kraken: ultrafast metagenomic sequence classification using exact alignments. Genome Biol. 15, R46 (2014).

29. Lu, J., Breitwieser, F. P., Thielen, P. \& Salzberg, S. L. Bracken: Estimating species abundance in metagenomics data. PeerJ 2017, (2017).

30. Jia, B. et al. CARD 2017: Expansion and model-centric curation of the comprehensive antibiotic resistance database. Nucleic Acids Res. 45, D566-D573 (2017).

31. Alcock, B. P. et al. CARD 2020: antibiotic resistome surveillance with the comprehensive antibiotic resistance database. Nucleic Acids Res. (2019) doi:10.1093/nar/gkz935.

32. BBMap: A Fast, Accurate, Splice-Aware Aligner (Conference) | OSTI.GOV. https://www.osti.gov/biblio/1241166.

33. Martínez, J. L., Coque, T. M. \& Baquero, F. What is a resistance gene? Ranking risk in resistomes. Nat. Rev. Microbiol. 13, 116-23 (2015).

34. Carpenter, B. et al. Stan: A probabilistic programming language. J. Stat. Softw. 76, (2017).

35. Vehtari, A., Gelman, A. \& Gabry, J. Practical Bayesian model evaluation using leave-one-out cross-validation and WAIC. Stat. Comput. 27, 1413-1432 (2017).

36. Yao, Y., Vehtari, A., Simpson, D. \& Gelman, A. Using Stacking to Average Bayesian Predictive Distributions (with Discussion). Bayesian Anal. 13, 917-1007 (2018).

37. R Core Team. R: A language and environment for statistical computing. (2018).

38. World Health Organisation. Prioritization of pathogens to guide discovery, research and development of new antibiotics for drug-resistant bacterial infections, including tuberculosis. https://www.who.int/medicines/areas/rational_use/prioritization-of-pathogens/en/ (2017).

39. Tacconelli, E. et al. Discovery, research, and development of new antibiotics: the WHO 
priority list of antibiotic-resistant bacteria and tuberculosis. Lancet Infect. Dis. 18, 318-327 (2018).

40. Kagia, N. et al. Carriage and Acquisition of Extended-spectrum $\beta$-Lactamase-producing Enterobacterales among Neonates Admitted to Hospital in Kilifi, Kenya. Clin. Infect. Dis. 69, 751-759 (2019).

685

41. Brooks, B. et al. Strain-resolved analysis of hospital rooms and infants reveals overlap between the human and room microbiome. Nat. Commun. 8, (2017). 


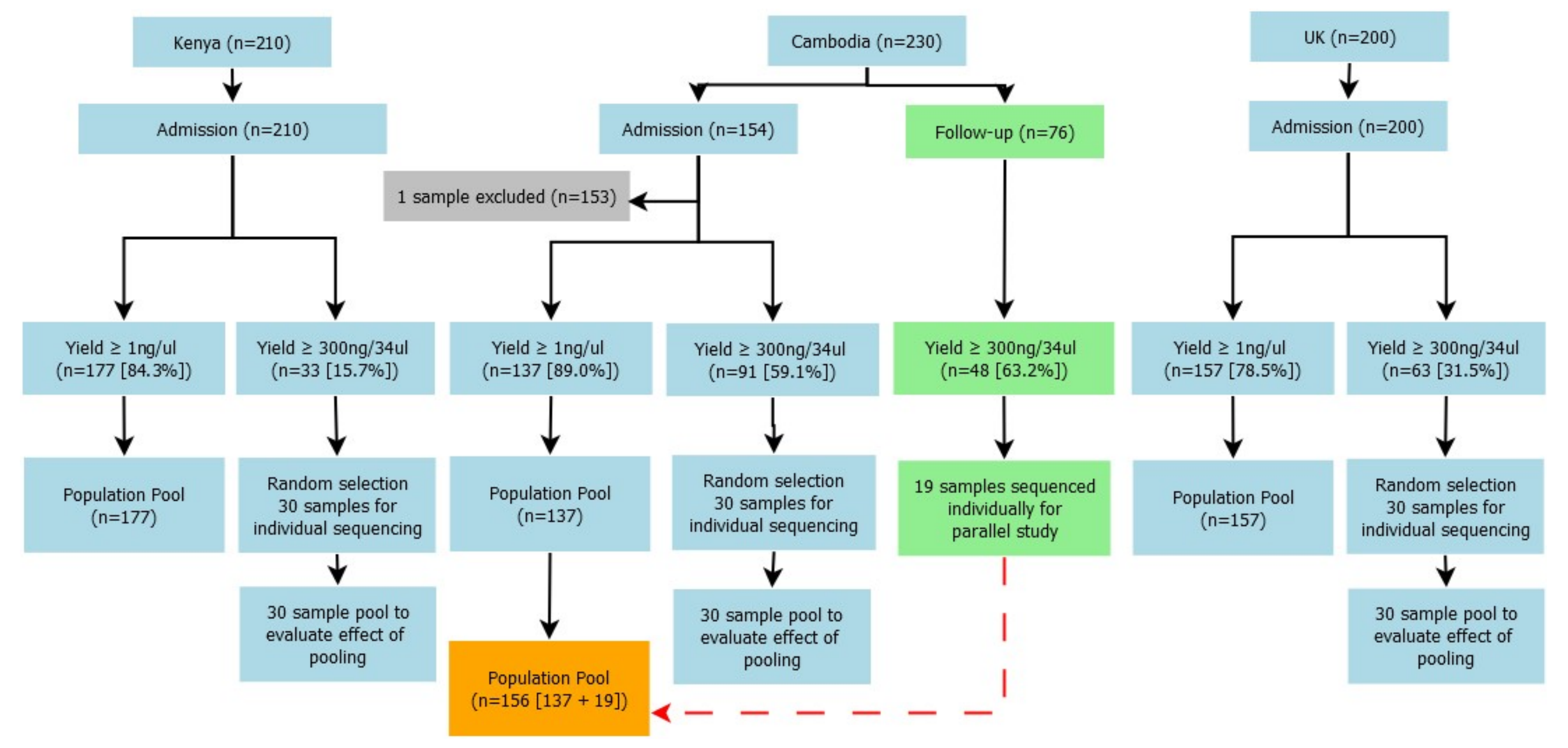


Population Pools

Enterobacterales Order \& Enterobacteriaceae Family

$A$

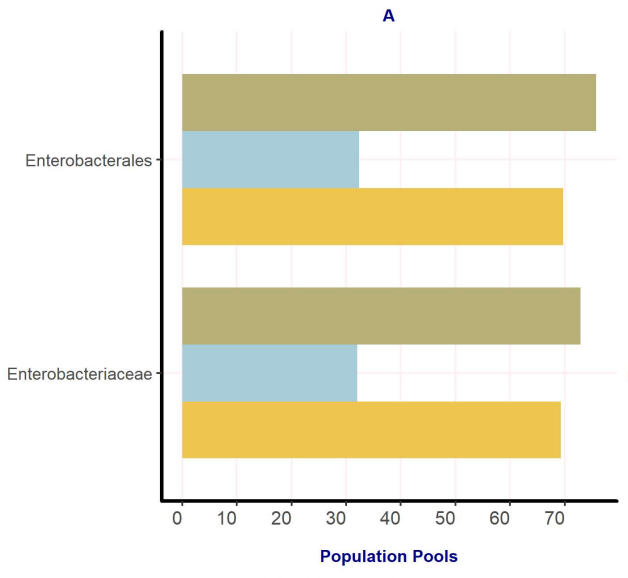

Other Enterobacterales Families

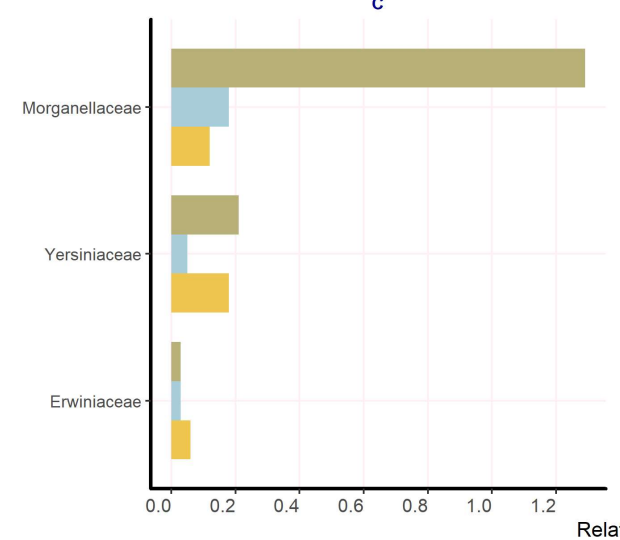

Blood \& Cerebrospinal Fluid Infections Enterobacterales Order \& Enterobacteriaceae Family
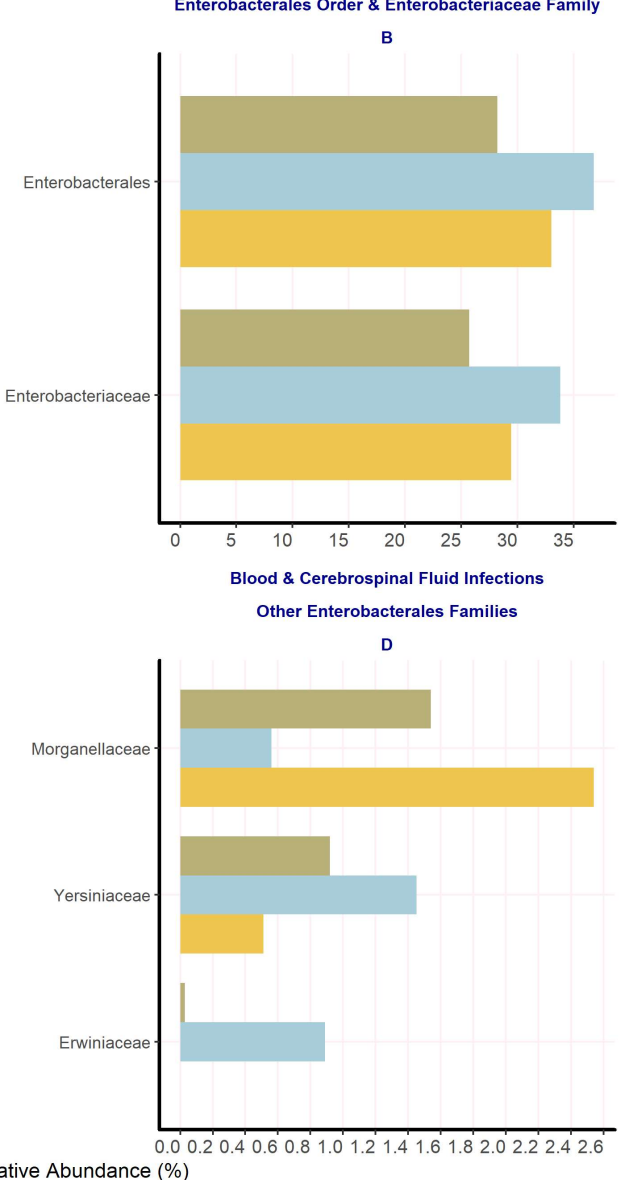

Population Pools

Main Enterobacterales Genera \& Species

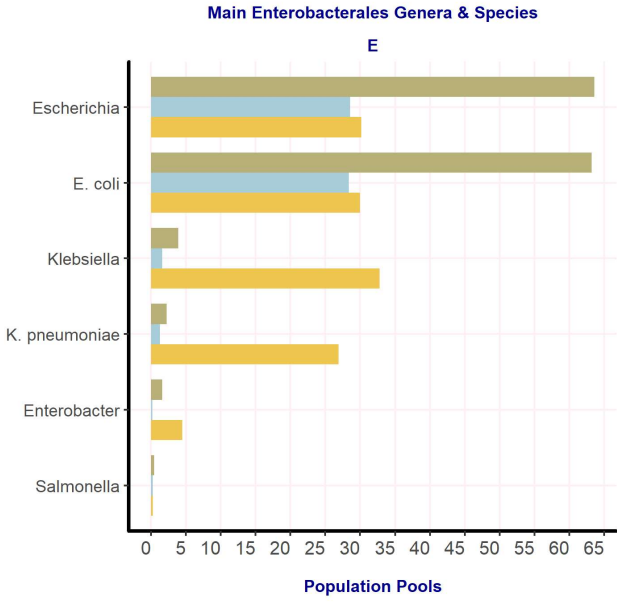

Other Enterobacterales Genera \& Species

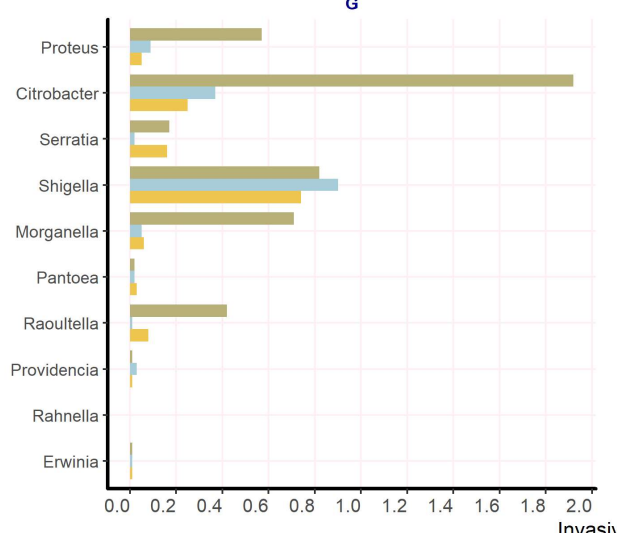

Blood \& Cerebrospinal Fluid Infections Main Enterobacterales Genera \& Species

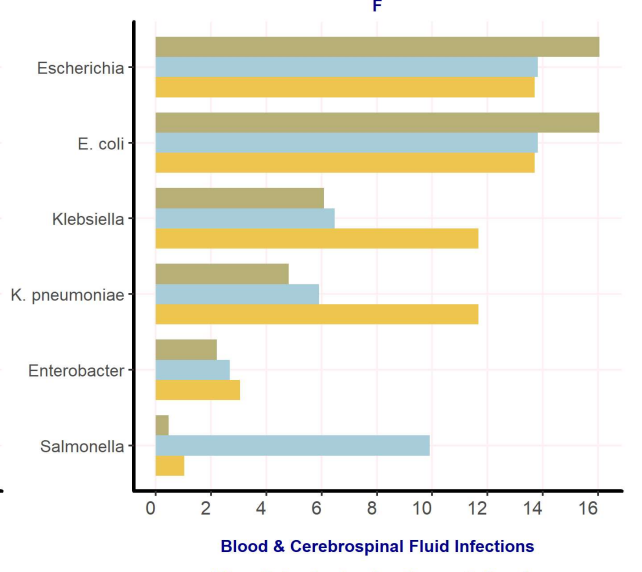
Other Enterobacterales Genera \& Species

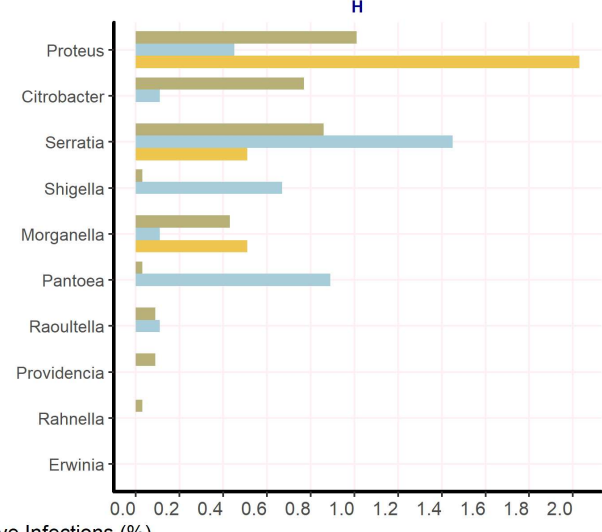

Setting $\square$ Cambodia $\square$ Kenya $\square$ UK 
A

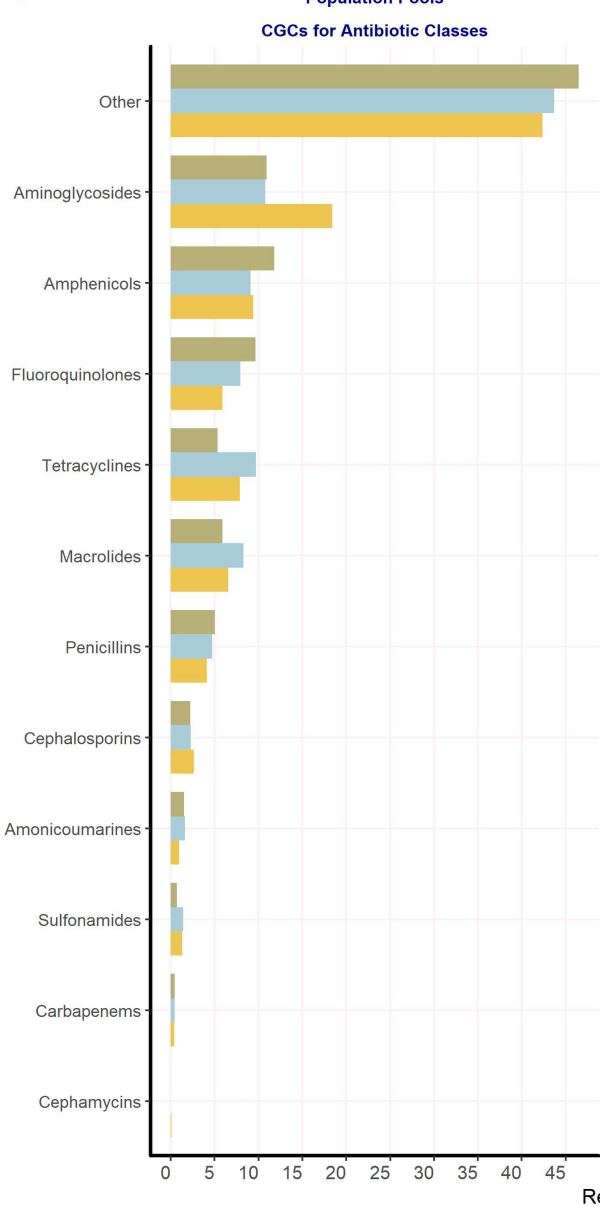

Population Pools

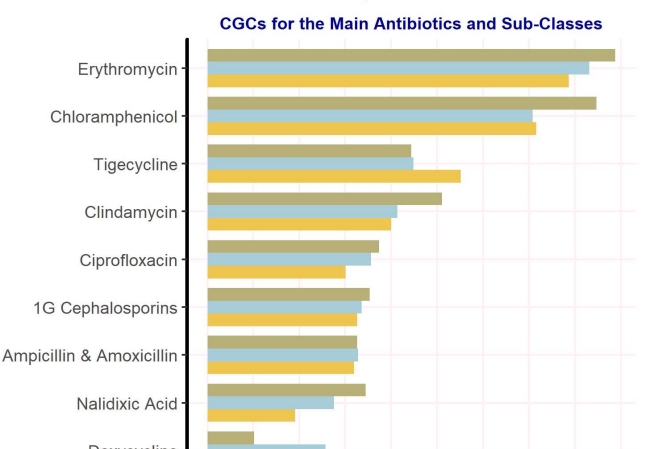

B

Population Pools

CGCs for Antibiotics in the Analysis

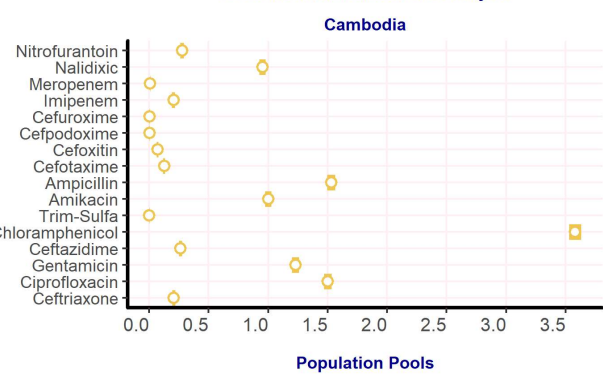

CGCs for Antibiotics in the Analysis

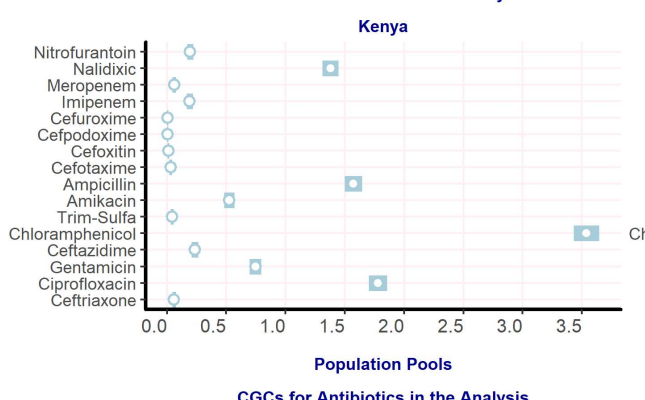

CGCs for Antibiotics in the Analysis

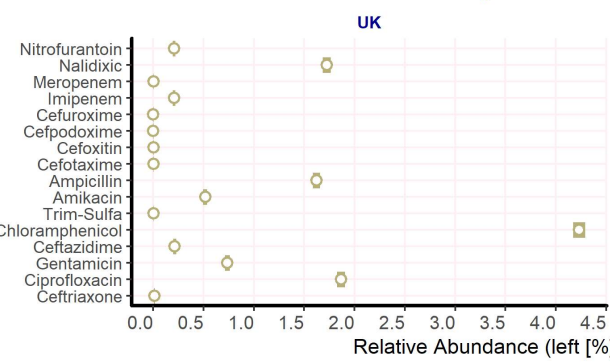

Blood \& Cerebrospinal Fluid Infections Enterobacterales Resistant Infections in the Analysis Cambodia

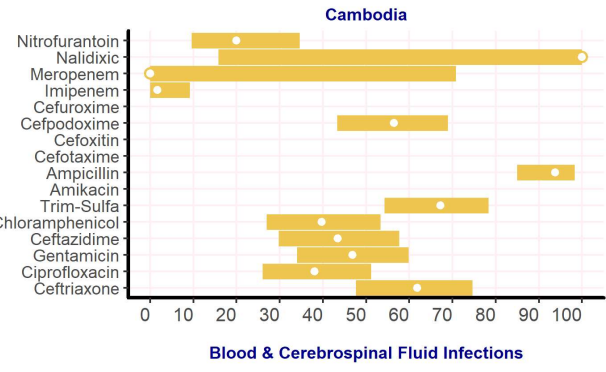
Enterobacterales Resistant Infections in the Analysis

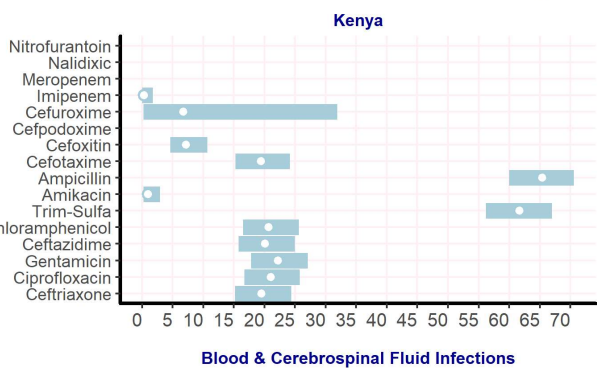

Fluid Infections Enterobacterales Resistant Infections in the Analysis

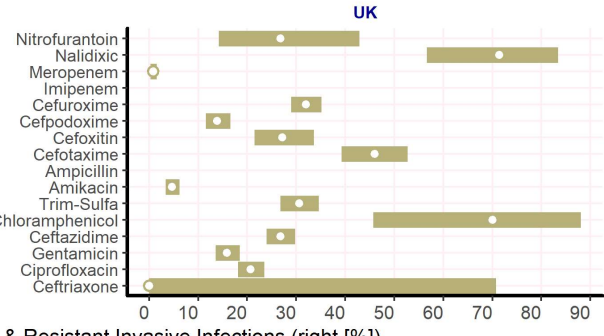
Relative Abundance (\%)

Setting $\square$ Cambodia $\square$ Kenya $\square$ UK 


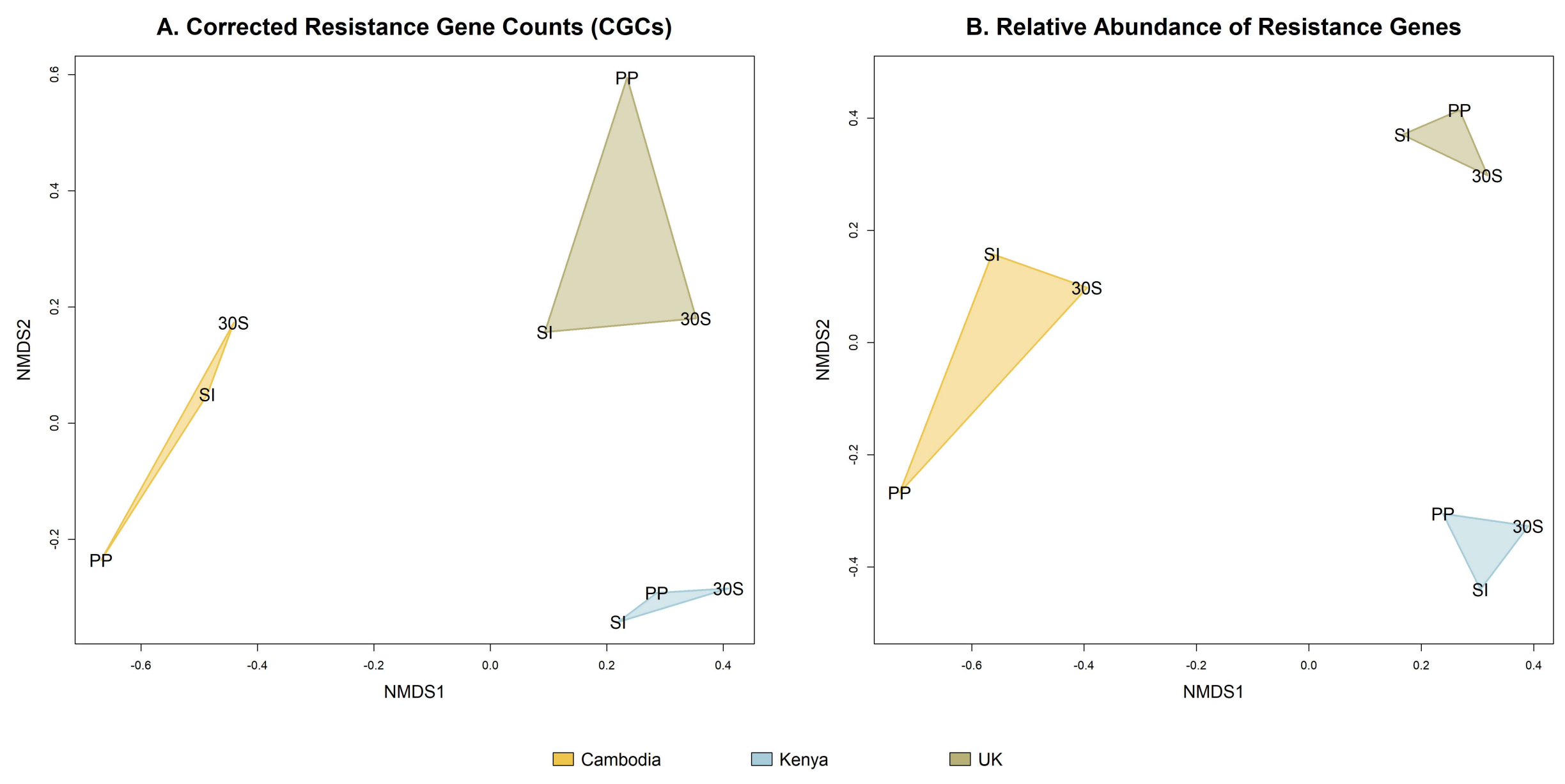




\section{Cambodia}

Amikacin (NT) Ampicillin (65)

Cefotaxime (NT)

Cefoxitin (NT)

Cefpodoxime (62)

Ceftazidime (53)

Ceftriaxone (55)

Cefuroxime (NT)

Chloramph (58)

Ciprofloxacin (63)

Gentamicin (62)

Imipenem (58)

Meropenem (3)

Nalidixic Acid (2)

Nitrofurantoin (45)

Trim-Sulfa (64)

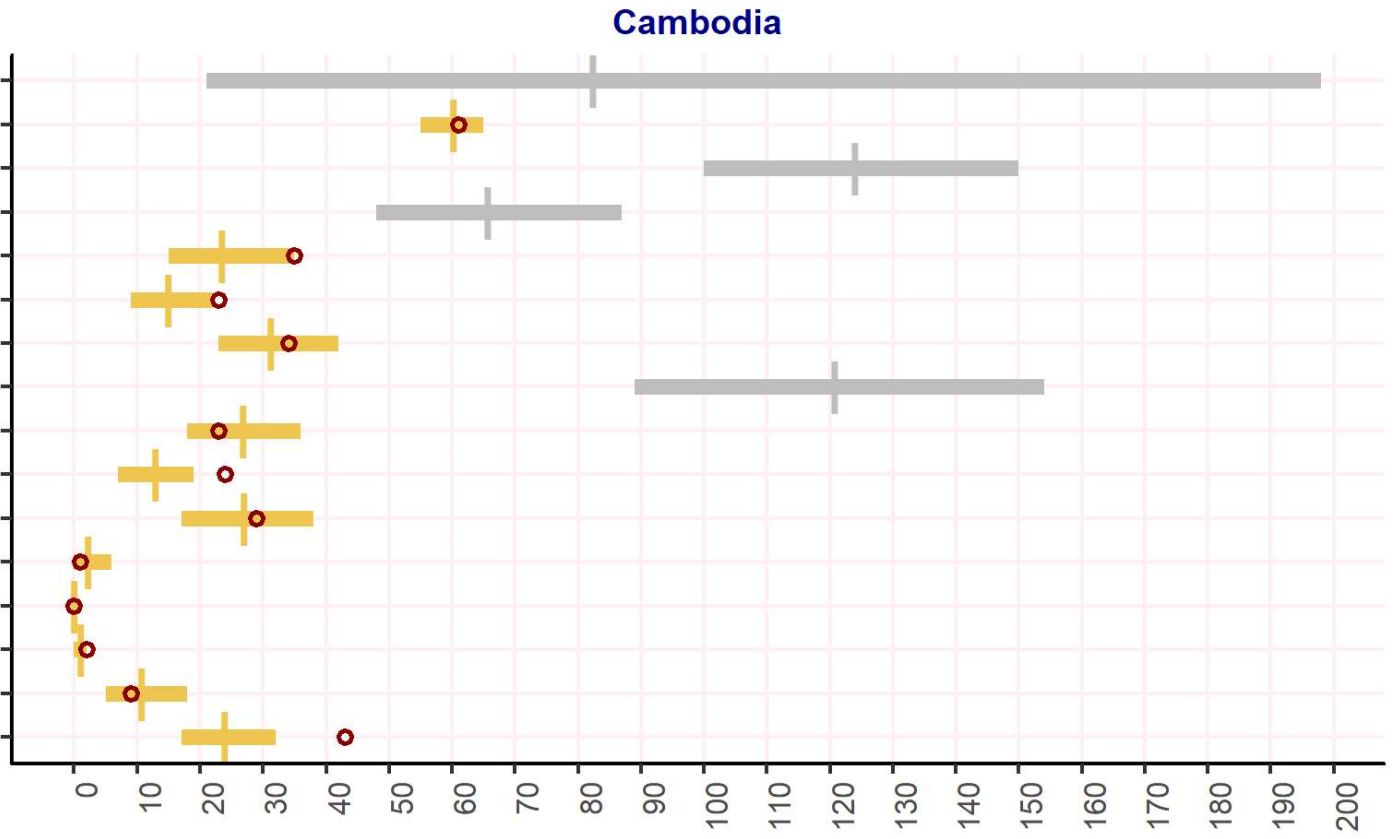

Amikacin (304

Ampicillin (324)

Cefotaxime (324)

Cefoxitin (306)

Cefpodoxime (NT)

Ceftazidime (308)

Ceftriaxone (308)

Cefuroxime (15)

Chloramph (323)

Ciprofloxacin (324)

Gentamicin (324)

Imipenem (311)

Meropenem (NT)

Nalidixic Acid (NT)

Nitrofurantoin (NT)

Trim-Sulfa (324)

\section{Kenya}

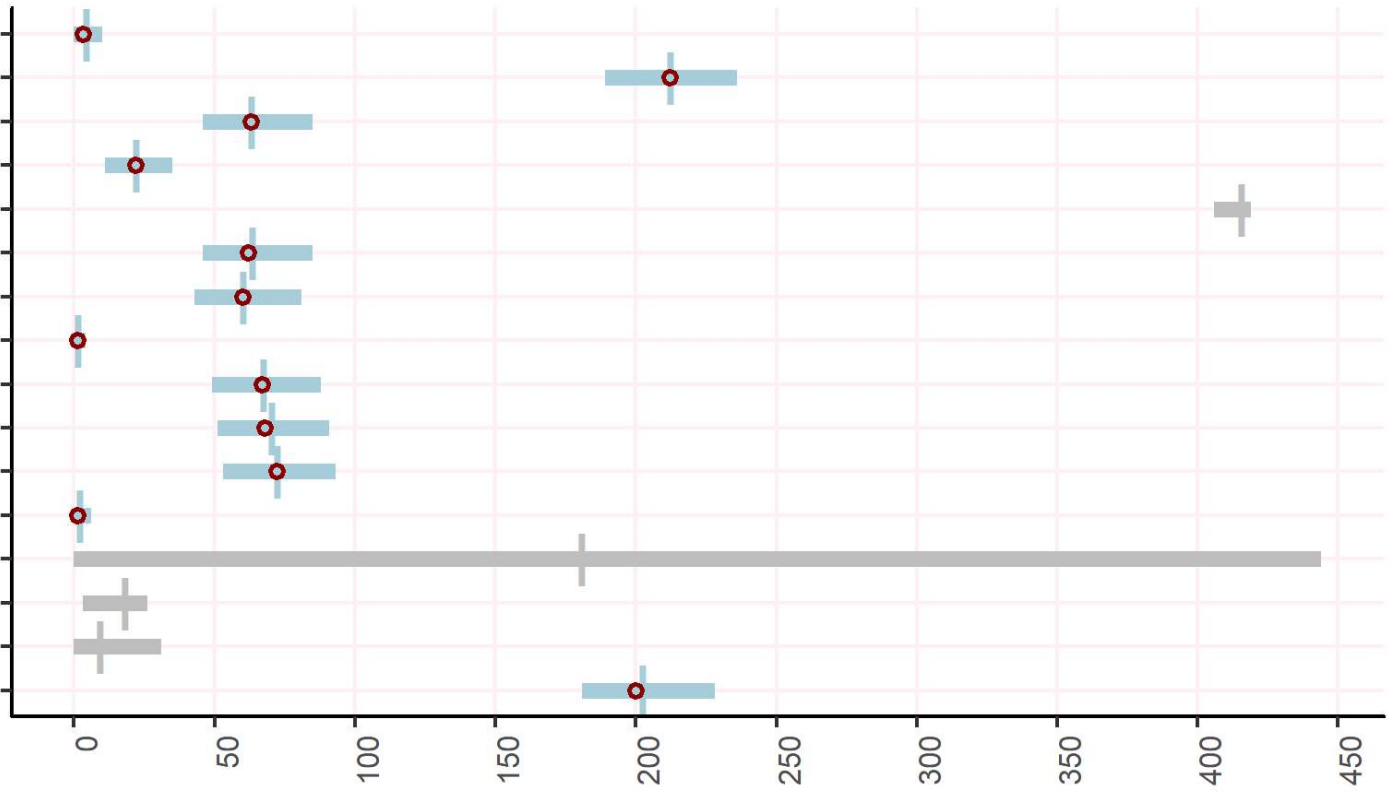

UK

Amikacin (903)

Ampicillin (NT)

Cefotaxime (224)

Cefoxitin (224)

Cefpodoxime (776)

Ceftazidime (906)

Ceftriaxone (3)

Cefuroxime (908)

Chloramph (20)

Ciprofloxacin (912)

Gentamicin (905)

Imipenem (NT)

Meropenem (908)

Nalidixic Acid (49)

Nitrofurantoin (41)

Trim-Sulfa (549)

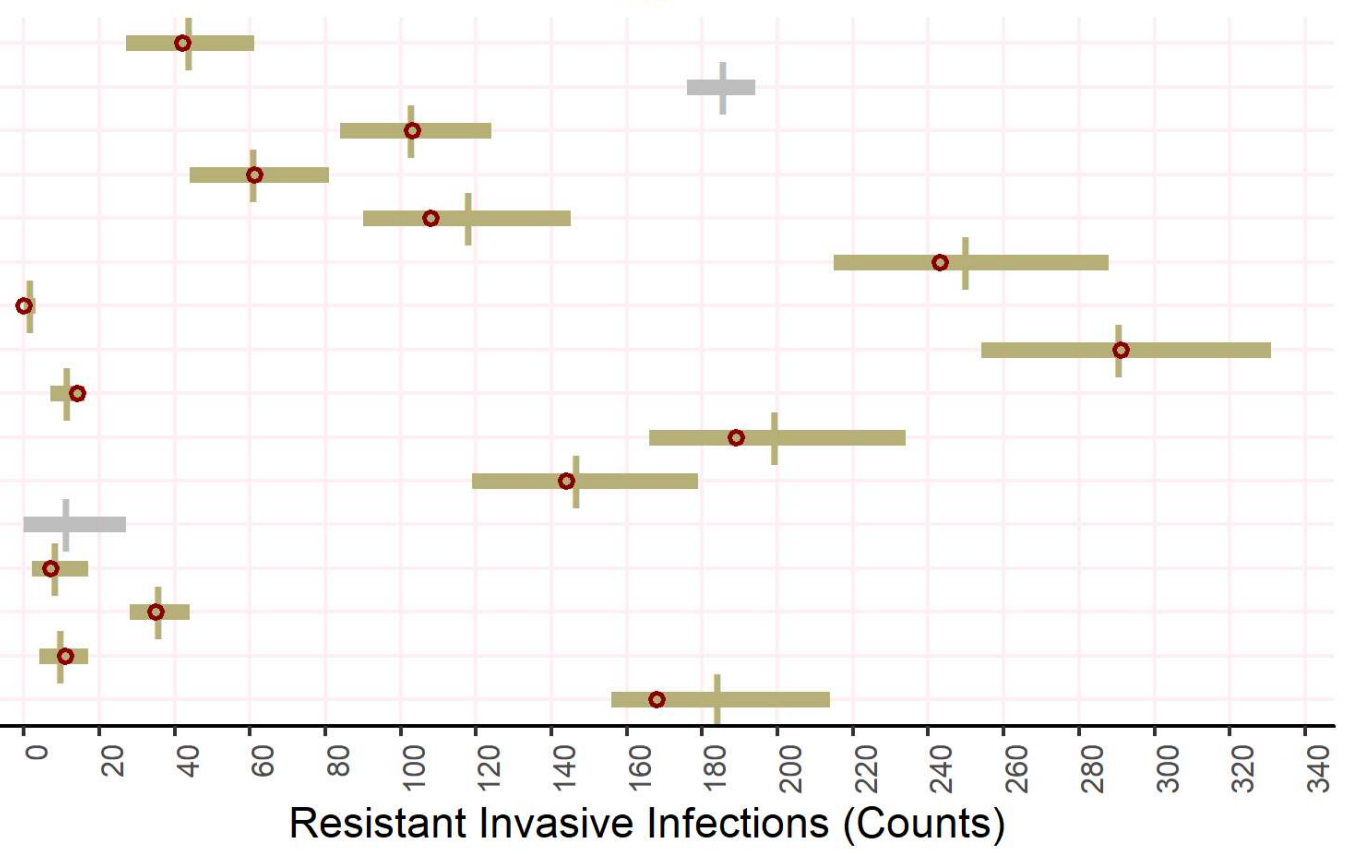


Blood \& Cerebrospinal Fluid Infections

All Genera

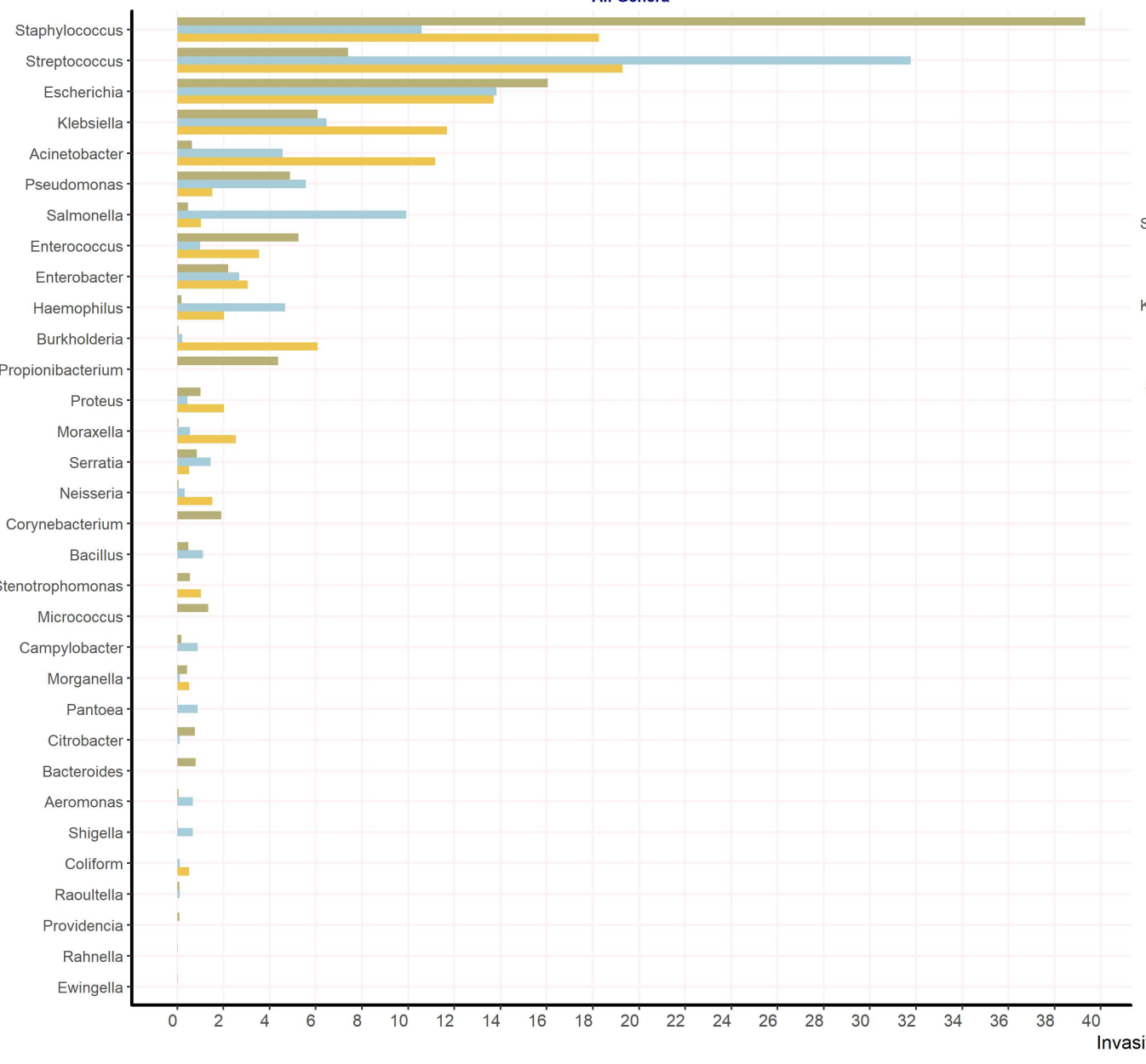

Blood \& Cerebrospinal Fluid Infections All Species

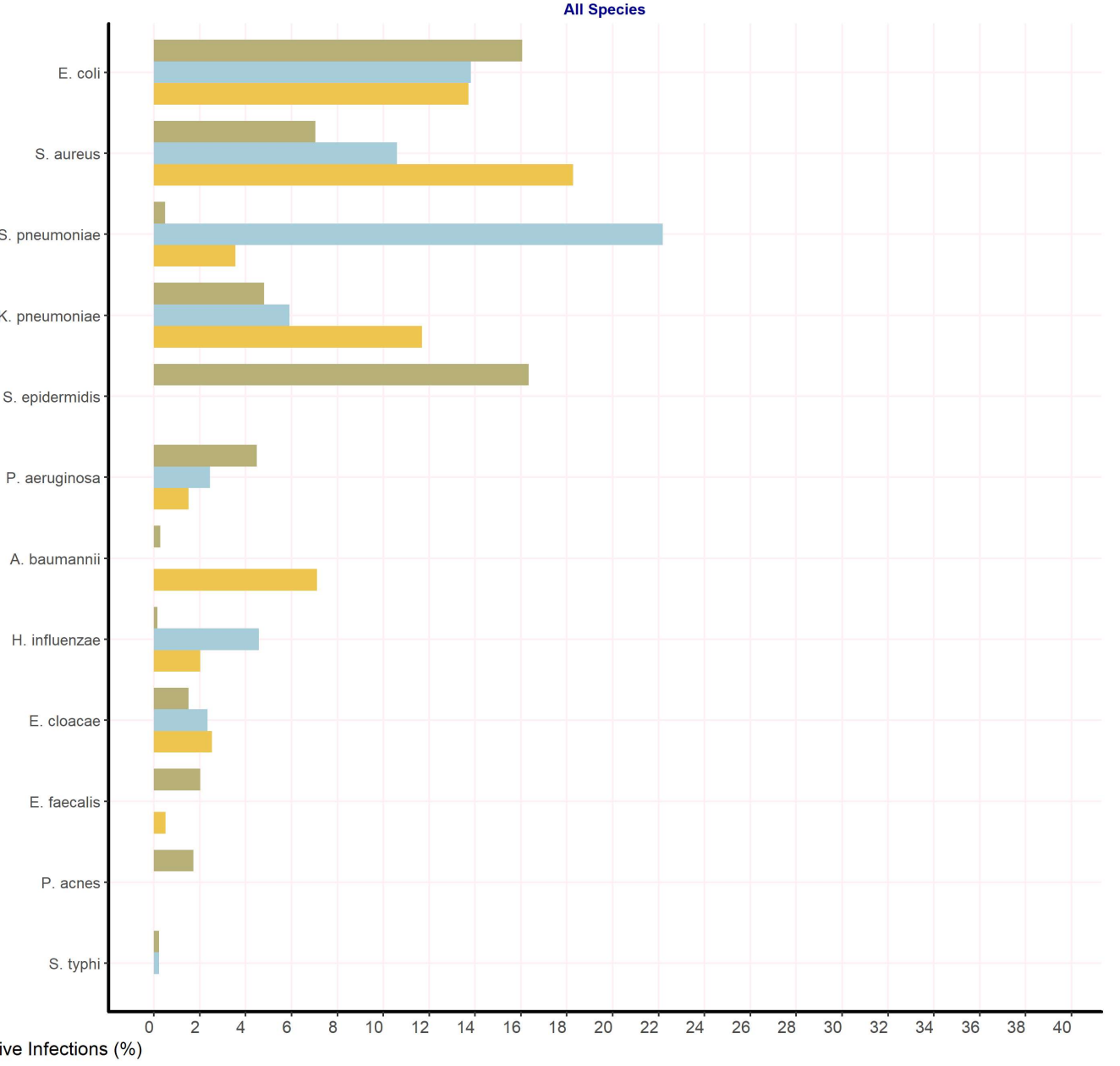

Setting Cambodia $\square$ Kenya UK 
bioRxiv preprint doi: https://doi.org/10.1101/2020.02.10.941930; this version posted February 11, 2020. The copyright holder for this preprint (which was not certified by peer review) is the author/funder, who has granted bioRxiv a license to display the preprint in perpetuity. It is made available under aCC-BY-NC-ND 4.0 International license.

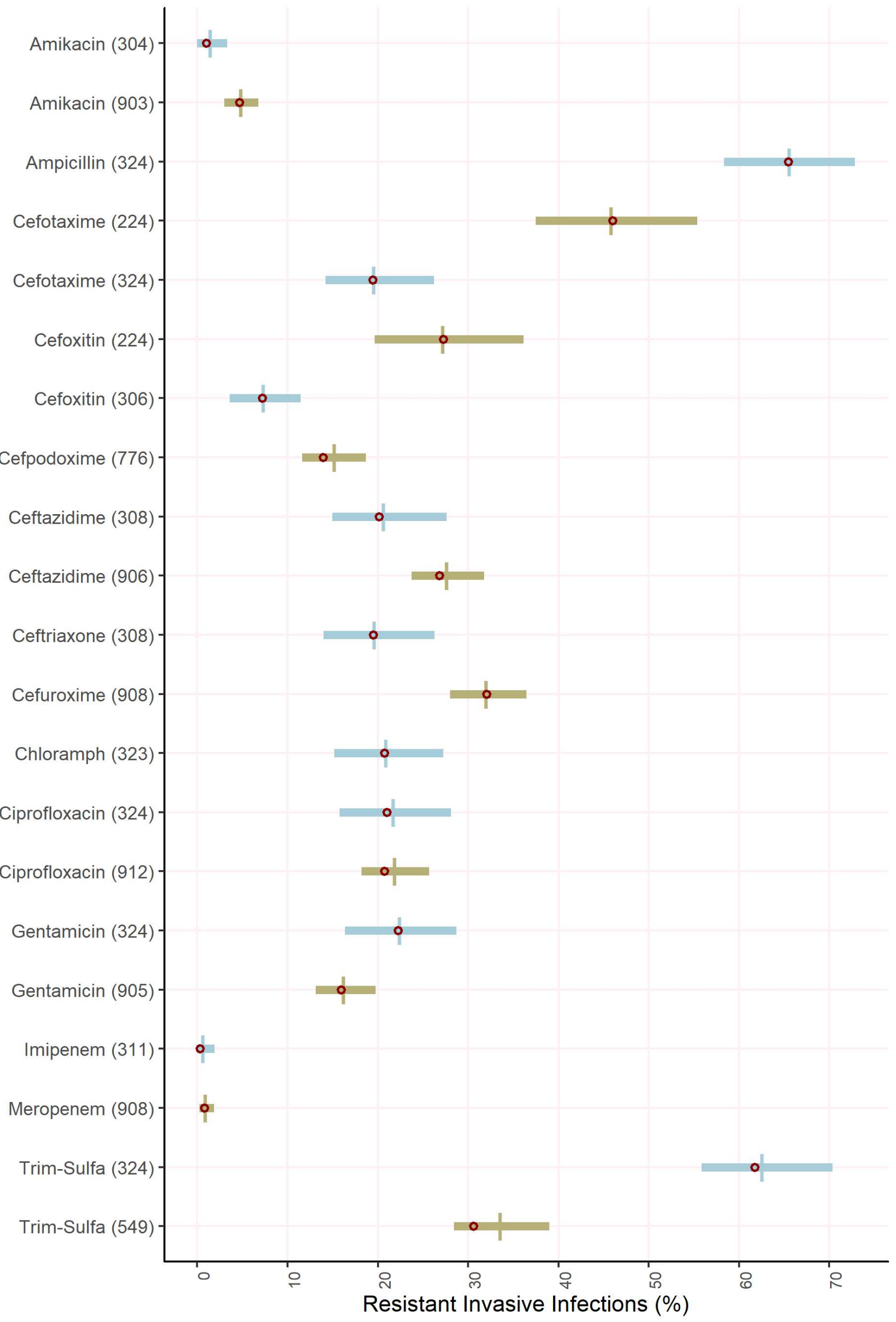

Setting Kenya UK 